\title{
Acute Loss-of-consciousness in Patient with Relapsing Candidemia and Bacteremia
}

\author{
Adnan Vural' ${ }^{1}$, Sevtap Arslan² (D), Timüçin Erol ${ }^{3}$ (D), Ahmet Çağkan Inkaya ${ }^{4}$ (D), Kader Karlı Oğuz² (D), \\ Ömrüm Uzun 4 (iD \\ 1 Anesthesiology and Reanimation Critical Care Unit, Hacettepe University School of Medicine, Ankara, Turkey \\ 2 Department of Radiology, Hacettepe University School of Medicine, Ankara, Turkey \\ 3 Department of Department of Ceneral Surgery, Hacettepe University School of Medicine, Ankara, Turkey \\ 4 Department of Infectious Diseases and Clinical Microbiology, Hacettepe University School of Medicine, Ankara, Turkey
}

A 43-year-old female patient underwent several abdominal surgeries including a liver hydatid cyst operation (34 years ago); Roux- $\mathrm{N}-\mathrm{Y}$ anastomosis plus hepaticojejunostomy plus cholecystectomy (27 years ago); and left salpyngectomy plus lower intestinal resection and end-to-end anastomosis (16 months ago). Biliary tract injury complicated the latest course of surgery which further necessitated numerous interventions to control biliary leakage. Eventually, she developed several enterocutaneous fistulae. She was fed through central venous catheter and jejeunostomy tube throughout her hospitalization and received treatments for multiple episodes of bloodstream infections caused by multidrug-resistant Acinetobacter baumannii, carbapenem-resistant Klebsiella pneumoniae (CRKP) and Morganella morganii, Candida albicans and Candida glabrata. While still hospitalized after 16-month of her hospitalization, one night the nurse on-call found her unconscious lying on the floor in her bedroom as her central venous catheter was disconnected from the line. Glasgow coma scale was 5 , thus she was intubated and resuscitated. Imaging studies were carried out.

Follow-up blood cultures remained sterile. The final diagnosis was cerebral air emboli. Neurologic condition of the patient further deteriorated. Eventually, she died one month after the event.

\author{
Corresponding Author: \\ Adnan Vural \\ E-mail: \\ dradnanvural@hotmail.com \\ Received: March 24, 2020 \\ Accepted: April 29, 2020 \\ Published: April 30, 2020

\section{Suggested citation:} \\ Vural A, Arslan S, Erol T, Inkaya \\ AÇ, Oğuz KK, Uzun Ö. Acute \\ Loss-of-consciousness in Patient \\ with Relapsing Candidemia \\ and Bacteremia. Infect Dis Clin \\ Microbiol 2020; 1: 52-53. \\ DOI: $10.36519 / \mathrm{idcm} .2020 .0002$
}




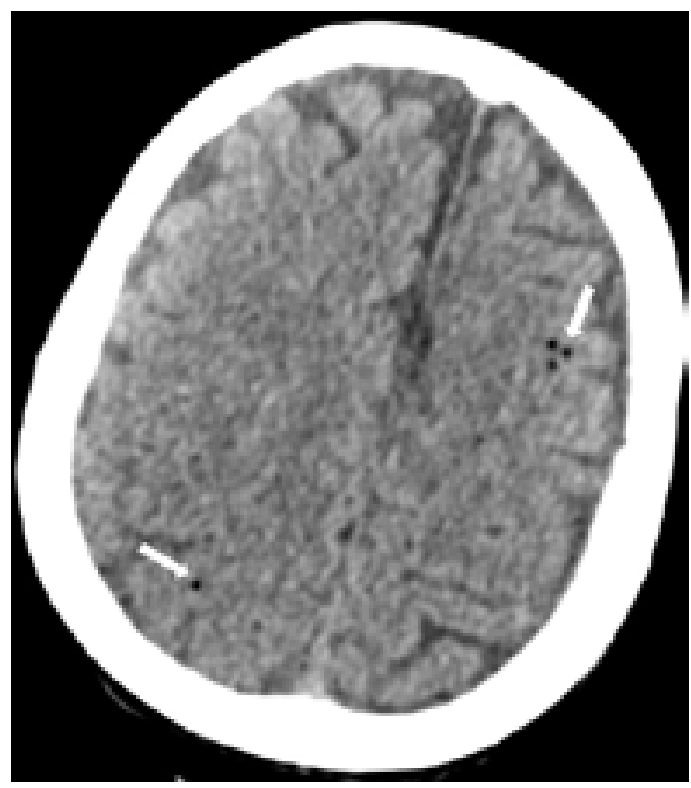

Figure 1.
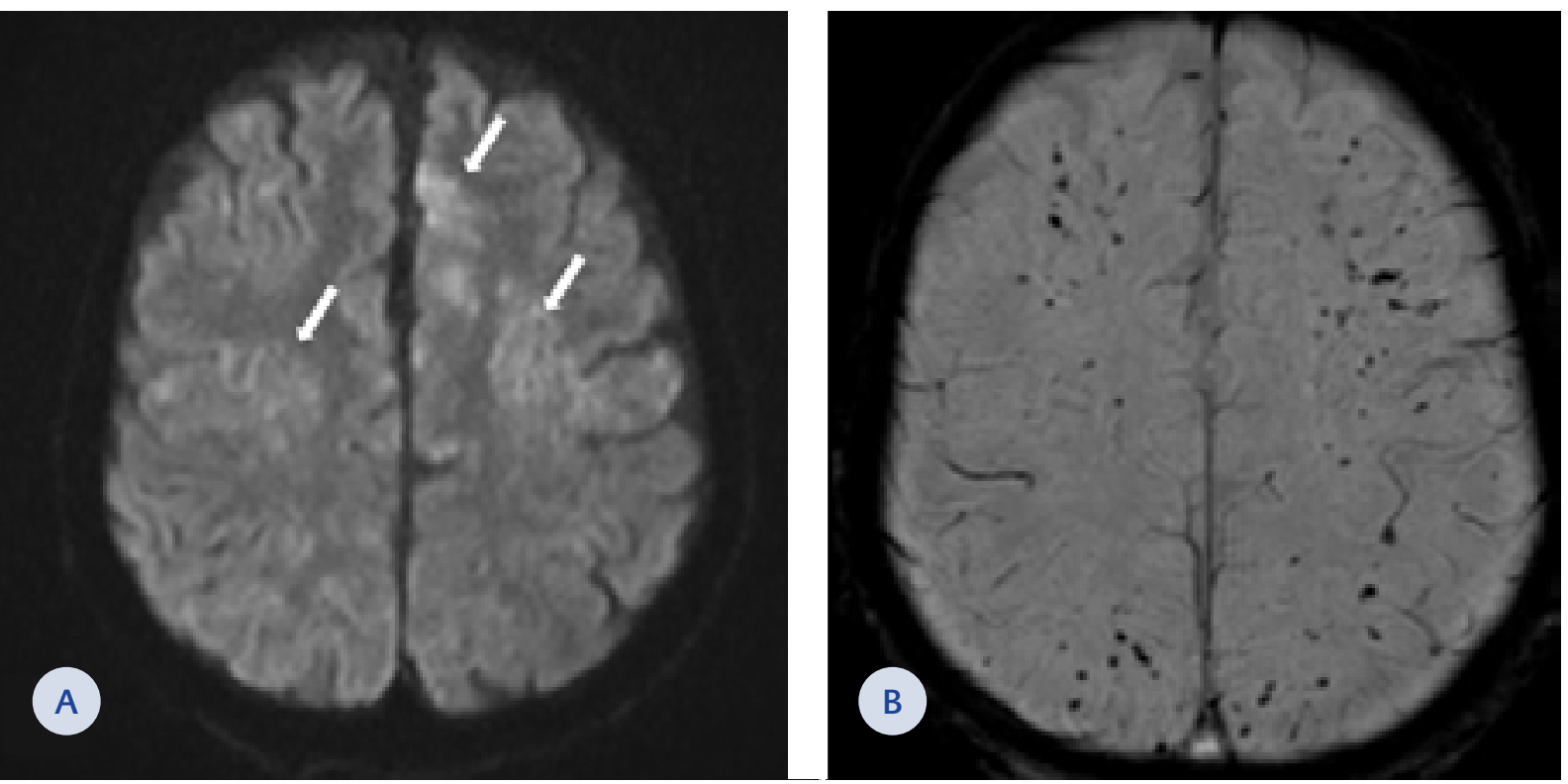

\section{Figure 2.}

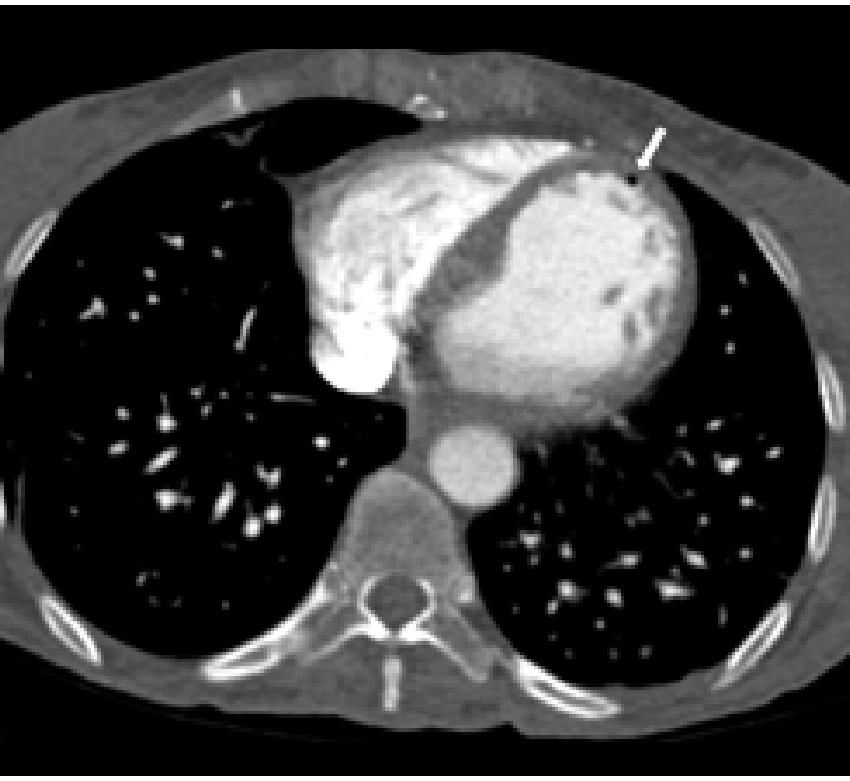

Figure 3. 